\title{
Classification of Defects in the Polarizer of Display Panels using the Convolution Neural Network (CNN)
}

\author{
Young Ha Won ${ }^{1}$, Hyonam $\mathrm{Joo}^{2}$, and Joon Seek Kim ${ }^{3}$
}

\begin{abstract}
In this paper, we propose a method to classify defects in the polarizer of display panels using the Convolution Neural Network (CNN). The defects (pol-defects) are produced while the polarizer is attached to the panel due to the physical contact. It is very important to detect such defects since they are one of the very critical factors that determine the lifetime of a display panel, especially that of the Organic Light Emitting Diode (OLED) type panels. The shapes of the pol-defects are irregular and the size of them varies a lot, which makes it difficult to even detect them from the image. Even more confusing is that there are defect-like objects which look quite similar to the real defects. Assuming that the regions which contain defects and/or defect-like objects are detected, an algorithm is developed to learn and classify the pol-defects from the remainder of the images using the CNN. A set of total 210 real images that consists of 70 background images, 70 defect-like objects, and 70 defects is collected and is used to teach the CNN. The designed CNN with proper parameter settings learns the defects well and shows the correct classification rate of $95 \%$.
\end{abstract}

Keywords - Convolutional Neural Network, Defect classification, Defect detection, Pol-defect.

\section{INTRODUCTION}

$\mathrm{R}$ ECENTLY, the Convolution Neural Network (CNN) has been successfully applied to pattern classification problems. Examples include the optical numeric recognition [1], the optical character recognition [1,2], the face recognition [3], the object and/or personal recognition [4], and the pedestrian recognition [5], etc. It is also applied to defect classification problem in Liquid Crystal Display (LCD) panels, such as the neural networks using BEP [6], a method using FFT [7], a method using SVD [8], a method using LS-SVM [9], and a method using a cumulative histogram [10].

Various defects occur in the manufacturing process of the Organic Light Emitting Diode (OLED) panel. They are black spots, uneven luminance, surface scratches, and defects on the polarizer (pol-defects), to name a few. Among them, the

Young Ha Won ${ }^{1}$ Dept. of Display Engineering, Hoseo University Asan-si, Chungnam, Korea(phone:+8210-4548-1678, e-mail: wonyoungha@gmail.com ) .

Hyonam $\mathrm{Joo}^{2}$, Dept. of Display Engineering, Hoseo University Asan-si, Chungnam, Korea (e-mail: hnjoo@hoseo.edu ).

Joon Seek Kim ${ }^{3}$ Dept. of Electronics Engineering, Hoseo University Asan-si, Chungnam, Korea (e-mail: joonskim@hoseo.edu ). pol-defect is the most critical one to find since it affects the lifetime of the OLED panel most. The shapes of the pol-defects are irregular and the size of them varies a lot, which makes it difficult to even detect them from the image. Even more confusing is that there are defect-like objects which look quite similar to the real defects.

While there exist quite a few researches done in detecting and classifying defects on LCD panels as mentioned before, there seems not many papers on detecting and classifying pol-defects on OLED panels.

The defect detection system should include a proper image acquiring unit and an algorithm to locate and classify defects. We assume that a set of images are taken from a defect detection system and the regions which contain objects of interest are located by some image processing algorithm (we will describe such a system in another paper). In this paper, a CNN is designed to distinguish the pol-defects from other pol-like objects and/or normal backgrounds. The designed CNN with proper parameter settings learns the defects well and shows the correct classification rate of $95 \%$.

\section{RELATED BACKGROUND}

\section{A. Types of the OLED Defects}

There are many different kinds of defects that occur during the process of manufacturing the OLED panels [11, 12]. Table 1 lists a few types of such defects and the environment which causes the defects to appear.

TABLE I

TYPES OF THE OLED DEFECTS [11]

\begin{tabular}{|c|c|}
\hline Type of defect & Causes in production process \\
\hline Dark Point & $\begin{array}{c}\text { Grow of organic polymer of ITO } \\
\text { panel, Array process of ITO panel }\end{array}$ \\
\hline Non-uniform luminance & Grow of organic polymer of ITO panel \\
\hline Surface scratch & Package, test process \\
\hline Insufficient rubber width & Package process \\
\hline Lack of color uniformity & $\begin{array}{l}\text { Immature of OLED display } \\
\text { technology }\end{array}$ \\
\hline
\end{tabular}


The algorithms for detecting and classifying these defects have been actively studied in other papers. An optical system for acquiring defective images was designed [11], and an algorithm was designed using the Independent Component Analysis (ICA) to detect defects in the background of patterned OLED panels [12].

\section{B. Convolutional neural Network}

The CNN was first proposed by Yann LeCun for zip code recognition [1]. The Lenet-5 proposed by LeCun is shown in the figure 1

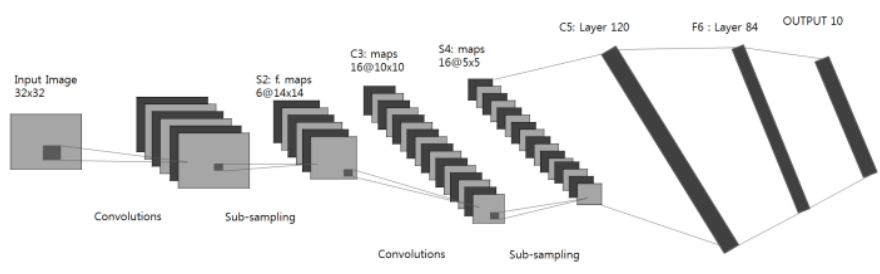

Fig. 1 Lenet-5 CNN

The size of the input image is $32 \times 32$ pixels, and the convolutions are performed with 6 kernels of size $5 \times 5$ pixels (C1). The resulting six $28 \times 28$ pixels images are sub-sampled by $2 \times 2$ max-pooling (S2). The sub-sampled images of size $14 \times 14$ pixels are selectively convolved again by six kernels of $5 \times 5$ pixels (C3), resulting in 16 images of size $10 \times 10$ pixels. They go through the same max-pooling as in $\mathrm{S} 2$, resulting in 16 images of size $5 \times 5$ pixels (S4). 120 features are selected to input to the fully connected neural networks with 84 hidden nodes followed by 10 output nodes.

The CNN gained high interest when LeCun's model won the first place in the 2012 ImageNet Large Scale Visual Recognition Challenge (ILSVRC) by a big margin over the $2^{\text {nd }}$ place winner [4]. Since then, the GoogleNet won the first place in ILSVRC in 2014.

The CNN, in general, consists of three layers. They are the convolution layer, the pooling layer, and the feedforward layer.

The convolution layer is a step to extract the main features from the input data. The parameters user can select include the size and number of the convolution kernel, and the kernel's center point, etc.

The pooling layer is a process of sub-sampling the features extracted from the convolution. The sub-sampling is typically done by the max-pooling and the average-pooling while the users can also design by their own. The pooling step selects a representative value within a kernel by selecting a maximum value (max-pooling) or an average value (average-pooling). The size of the kernel is again one of the user-selectable parameter.

The number of convolution and pooling layer pairs can be selected by a user.

The feedforward layer has the same structure as the conventional neural network. The input to this layer is the either the output of the convolution layer or the output of the pooling layer.

\section{INPUT IMAGE PREPARATION}

The images containing pol-defects and pol-like objects are obtained by an image acquisition system and by an algorithm that locates such regions. The images of background without any suspicious objects are also collected. Table 2 shows the images used in the design of our CNN.

TABLE I

IMAGES USED IN THE EXPERIMENT

\begin{tabular}{|c|c|}
\hline & Number of images \\
\hline Background & 70 \\
\hline Defect-like object & 70 \\
\hline Defect & 70 \\
\hline Total & 210 \\
\hline
\end{tabular}
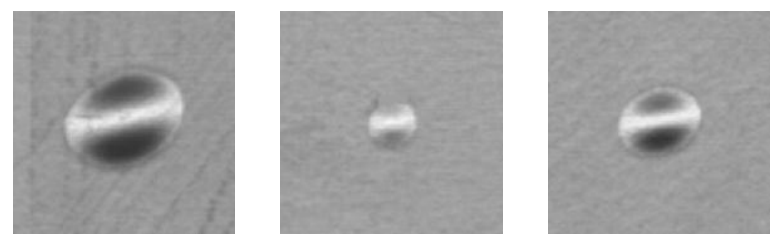

Fig. 2 Defect-like object images
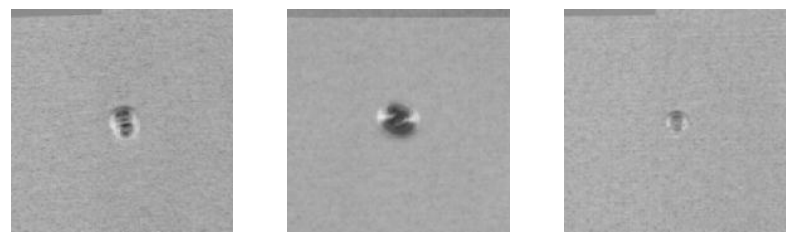

Fig. 3 Pol-defect images

Fig. 2 and fig 3 show an example images of defect-like objects and pol-defects, respectively. The sizes of these images are 256x256 pixels. It is assumed that these images are located and the Region of Interest (ROI) are selected from the original large image of possibly $9 \mathrm{~K}$ by $5 \mathrm{~K}$ size. The algorithm of locating these ROIs is not described in this paper and will be published in a separate paper.

Figs 4, 5, and 6 show the normalized images of size $64 \times 64$ pixels of the background, defect-like objects, and pol-defects, respectively. The pol-defects and the defect-like objects in Figs. 2 and 3 are put at the center of the image and the twice of both the width and the height of the objects are cut out from the original Figs. 2 and 3. Then they are cubic interpolated and are re-sized into $64 \times 64$ pixel images to make the so-called normal image. These normal images of 70 pixels each as in Table 1 are used to train and test the $\mathrm{CNN}$ in our application.

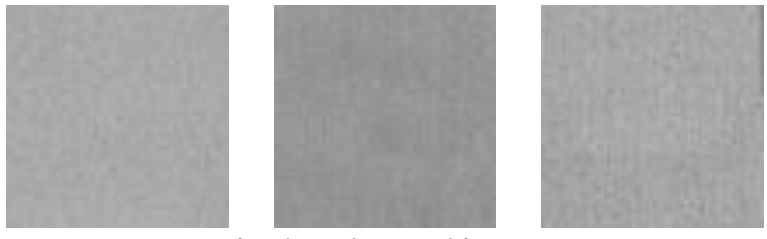

Fig. 4 Background images 

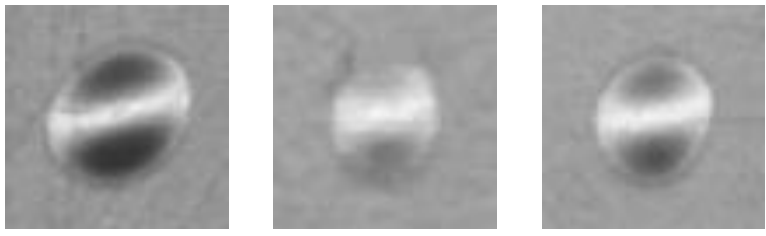

Fig. 5 Normalized defect-like object images
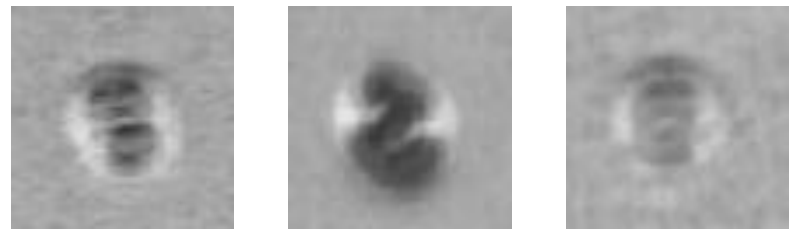

Fig. 6 Normalized pol-defect images

\section{DESIGN OF THE CNN}

Three CNNs are designed with the parameters set as shown in Table 3. All of them use 2 feedforward layers.

TABLE III

PARAMETERS OF THE DESIGNED CNNS

\begin{tabular}{|c|c|c|c|}
\hline \multirow{2}{*}{$\begin{array}{l}\text { Experiment } \\
\text { number }\end{array}$} & \multicolumn{3}{|c|}{ Layers } \\
\hline & Type & Size & $\begin{array}{c}\text { Number } \\
\text { of } \\
\text { kernels }\end{array}$ \\
\hline \multirow{6}{*}{1} & Convolution layer & 17 & 3 \\
\hline & Pooling layer & 2 & \\
\hline & Convolution layer & 5 & 2 \\
\hline & Pooling layer & 2 & \\
\hline & Convolution layer & 3 & 2 \\
\hline & Pooling layer & 2 & \\
\hline \multirow{4}{*}{2} & Convolution layer & 17 & 6 \\
\hline & Pooling layer & 2 & \\
\hline & Convolution layer & 9 & 2 \\
\hline & Pooling layer & 2 & \\
\hline \multirow{2}{*}{3} & Convolution layer & 33 & 6 \\
\hline & Pooling layer & 16 & \\
\hline
\end{tabular}

The convolution layers consist of three kernels of size $17 \times 17$ pixels, two kernels of size $5 \times 5$ pixels, and two kernels of size $3 \times 3$ pixels. All three pooling layers are max-pooling type kernels of size $2 \times 2$ pixels. The result of the last max-pooling layer is input to the feedforward layer. The schematic of the CNN in experiment 1 is shown in Fig. 7.

The experiment 2 and 3 are also designed as in Table 3. All three CNNs are trained in 1000 iterations and different learning rates are used to measure the performance.

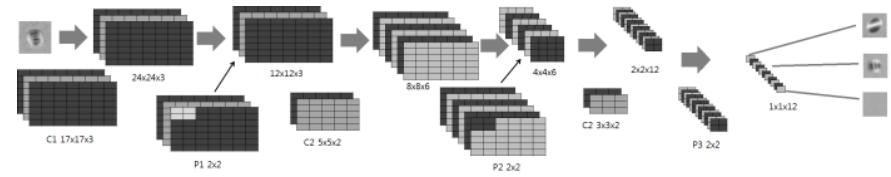

Fig. 7 Schematic of the Experiment 1

\section{EXPERIMENTAL RESULT}

Two types of tests are performed to measure the performance of the designed CNNs.

First, all the 210 images are used to train the specified CNNs and the same 210 images are used as test images. Table 4 shows the result of the first experiment with 1000 training iterations each.

TABLE IV

UNITS FOR MAGNETIC PROPERTIES

\begin{tabular}{|c|c|}
\hline Experiment number & Accuracy $(\%)$ \\
\hline 1 & 97 \\
\hline 2 & 98 \\
\hline 3 & 94 \\
\hline
\end{tabular}

To see how the designed CNNs are learning the problem, the error rates are measured at each steps of iteration and are plotted as in Figs. 8, 9, and 10. We only show the iteration step up to 1000 in these figures. In each of the figures, the error rates of 3 different learning rates w.r.t. the iteration steps are plotted for comparison. They are $0.0005,0.001$ and 0.0015 .

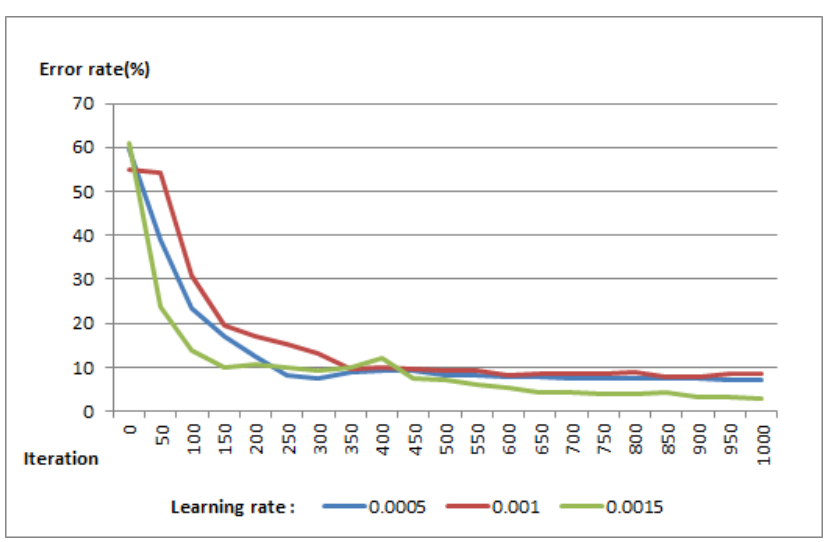

Fig. 8 Result of the experiment 1 with 3 learning rate

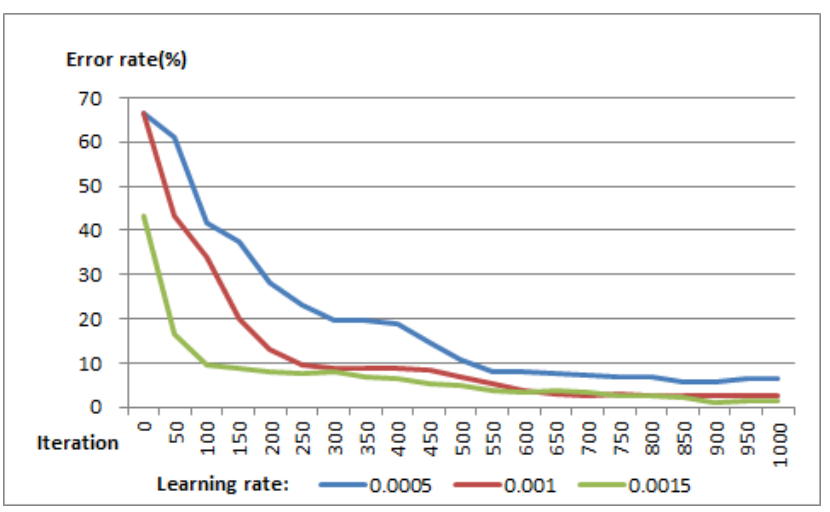

Fig. 9 Result of the experiment 2 with 3 learning rate 


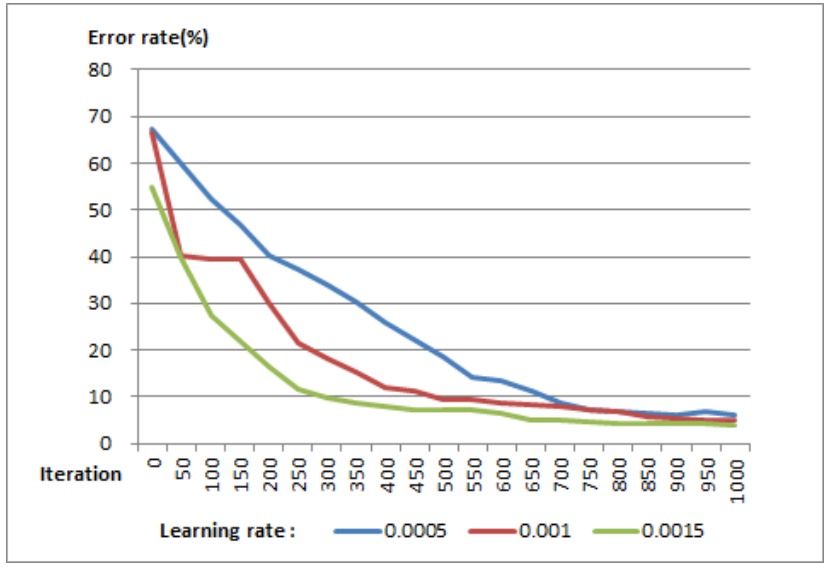

Fig.10 Result of the experiment 3 with 3 learning rate

Second, in another experiment ("leave one out"), we exclude one image from the 70 images each, and the remaining 3 sets of 69 images are used to train the CNNs. The learning rate of 0.0005 is used for this experiment. After the training is finished, the one left image each from the 3 sets of the original input image are classified by the trained CNNs. As shown in Table 5, with 1000 iteration steps, the experiment 2 performs well compared to the other experiments.

TABLE $\vee$

\begin{tabular}{|c|c|}
\hline CLASSIFICATION RESULT OF LEAVE-ONE-OUT EXPERIMENT \\
\hline $\begin{array}{c}\text { Experiment } \\
\text { number }\end{array}$ & Accuracy $(\%)$ \\
\hline 1 & 65.7 \\
\hline 2 & 94.7 \\
\hline 3 & 90.4 \\
\hline
\end{tabular}

TABLE VI

CONFUSION MATRIX OF THE EXPERIMENT 2

\begin{tabular}{|c|c|c|c|}
\hline Result Label & defect & $\begin{array}{c}\text { defect-lik } \\
\text { e object }\end{array}$ & Background \\
\hline defect & 65 & 3 & 2 \\
\hline defect-like object & 2 & 66 & 0 \\
\hline background & 3 & 1 & 68 \\
\hline Accuracy (\%) & 93 & 94 & 97 \\
\hline
\end{tabular}

Table. 6 is the confusion matrix of the experiment 2 which shows good classification accuracy. With more ground truth data, the CNN can be more accurately designed.

\section{CONCLUSION}

A method to classify defects in the polarizer of display panels using the Convolution Neural Network is proposed. Careful design of the CNN results in a good classification accuracy with only a few samples of ground truth images. Experiments show that with a set of total 210 real images that consists of 70 background images, 70 defect-like objects, and 70 defects, the
CNNs can be trained to give almost $95 \%$ classification accuracy.

\section{REFERENCES}

[1] Y. LeCun, L. Bottou, Y. Bengio, and P. Haffner."Gradient-based learning applied to document recognition", Proceedings of the IEEE, vol. 86, pp. 2278-2324, November 1998.

[2] Dan Claudiu Cires, an and Ueli Meier and Luca Maria Gambardella and J"urgen Schmidhuber, "Convolutional Neural Network Committees For Handwritten Character Classification", 2011 International Conference on Document Analysis and Recognition

[3] Christophe Garcia and Manolis Delakis, "Convolutional Face Finder : A Neural Architecture for Fast and Robust Face Detection", IEEE TRANSACTIONS ON PATTERN ANALYSIS AND MACHINE INTELLIGENCE, VOL. 26, NO. 11, NOVEMBER 2004

[4] A. Krizhevsky, I. Sutskever, and G. E. Hinton. Imagenet classification with deep convolutional neural networks. In Advances in neural information processing systems, 2012.

[5] Xuan-Phung Huynh, Yong-Guk Kim, "Deep Learning-based Multiple Pedestrians Detection-Tracking Framework",The HCI Society of Korea,2016.1, 55-60 (6 pages)

[6] Jung-Hwan Ko. "LCD Defect Detection using Neural-network based on BEP." The Institute of Electronics Engineers of Korea - IE 48(2), 2011.6, 26-31 (6 pages)Another reference

[7] Jun-Hyung Ha, Dong-In Yu, Young-Beom Jang. "LCD Mura Detection Using FFT"THE INSTITUTE OF ELECTRONICS ENGINEERS OF KOREA. 2009.7, 969-970 (2 pages)

[8] Jung-Hwan Ko, Won-Seok Lee, Young-Jin Won. "Adaptive defect pattern algorithm for LCD surface inspection". The Institute of Electronics Engineers of Korea -2010.6, 1787-1788 (2 pages)

[9] Ho-Hyung Choi, Gun-Hee Lee, "A Defect Inspection Method in TFT-LCD Panel Using LS-SVM" Ja-Geun Kim. . Korean Institute of Intelligent Systems 2009.12, 852-859 (8 pages)

[10] SeungMin Lee, Kil-Houm Park. "STD Defect Detection Algorithm by Using Cumulative Histogram in TFT-LCD Image." Journal of Korea Multimedia Society 19(8), 2016.8, 1288-1296 (9 pages)

[11] D B Perng, Y C Chen, M K Lee, "A novel AOI system for OLED panel inspection",7th International Symposium on Measurement Technology and Intelligent Instruments $\odot$ 2005, Journal of Physics: Conference Series 13 (2005) 353-356

[12] Zhiliang Wang, Jian Gao*, Chuanxia Jian,Yu Cen and Xin Chen, "OLED Defect Inspection System Development through Independent Component Analysis",TELKOMNIKA, Vol.10, No.8, December 2012, pp. 2309 2319

This work was supported by Industrial Human Resources and Skill Development Program (N0001415, Display Expert Training Project for Advanced Display equipment and components engineer) funded by the Ministry of Trade, Industry \& Energy (MOTIE, Korea) 